Supporting information for:

\title{
Stepwise Photocleavage of Two C-O Bonds of 1,8-Bis[(4-benzoylphenoxy)-methyl]naphthalene with Three-step Excitation using Three-color Three-laser Flash Photolysis
}

Xichen Cai, Masanori Sakamoto, Michihiro Hara, Sachiko Tojo, Akihiko Ouchi, " Kiyohiko Kawai,

Masayuki Endo, Mamoru Fujitsuka, and Tetsuro Majima

"The Institute of Scientific and Industrial Research (SANKEN), Osaka University, Mihogaoka 8-1, Ibaraki, Osaka 567-0047, Japan. $\quad$ "National Institute of Advanced Industrial Science and Technology, 1-1-1, Higashi, Tsukuba, Ibaraki 305-8565, Japan.

\section{Three-color three-laser flash photolysis of 1-(BPO-CH2) Np}

The experimental results of three-color three-laser photolysis of 1-(BPO- $\left.\mathrm{CH}_{2}\right) \mathrm{Np}$ are shown in Figure S1 A, B.

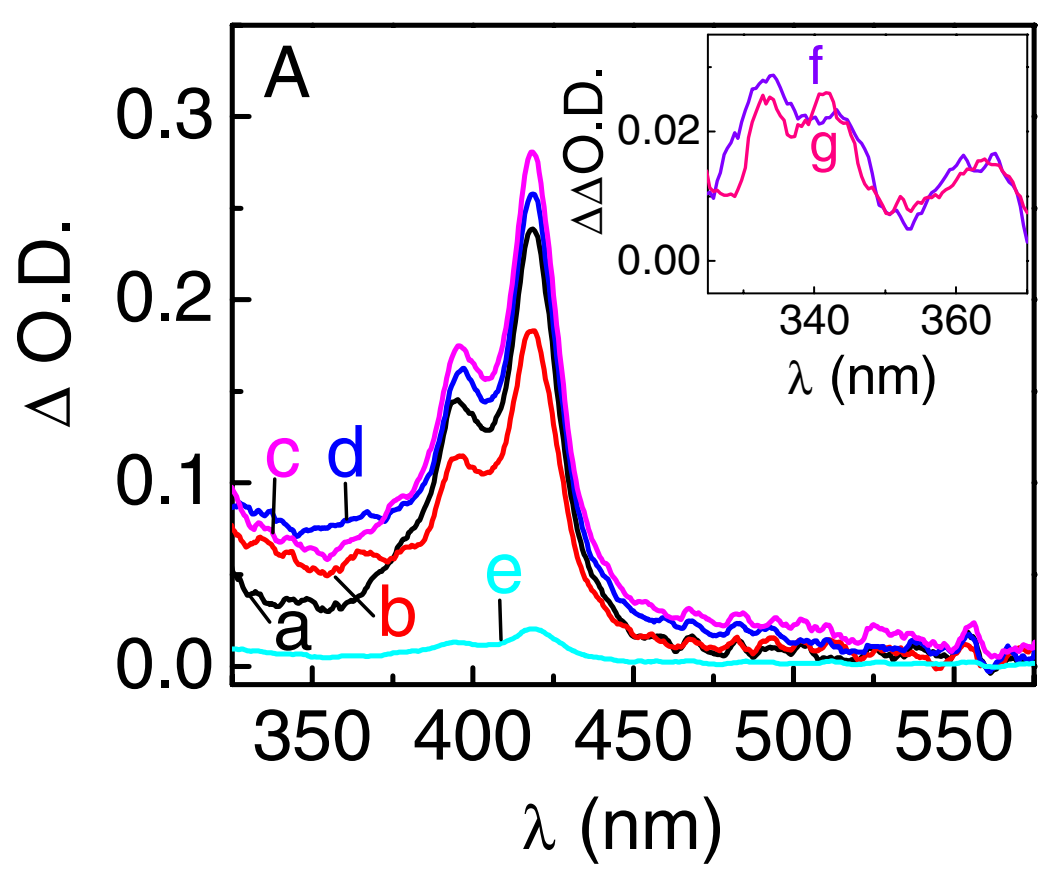




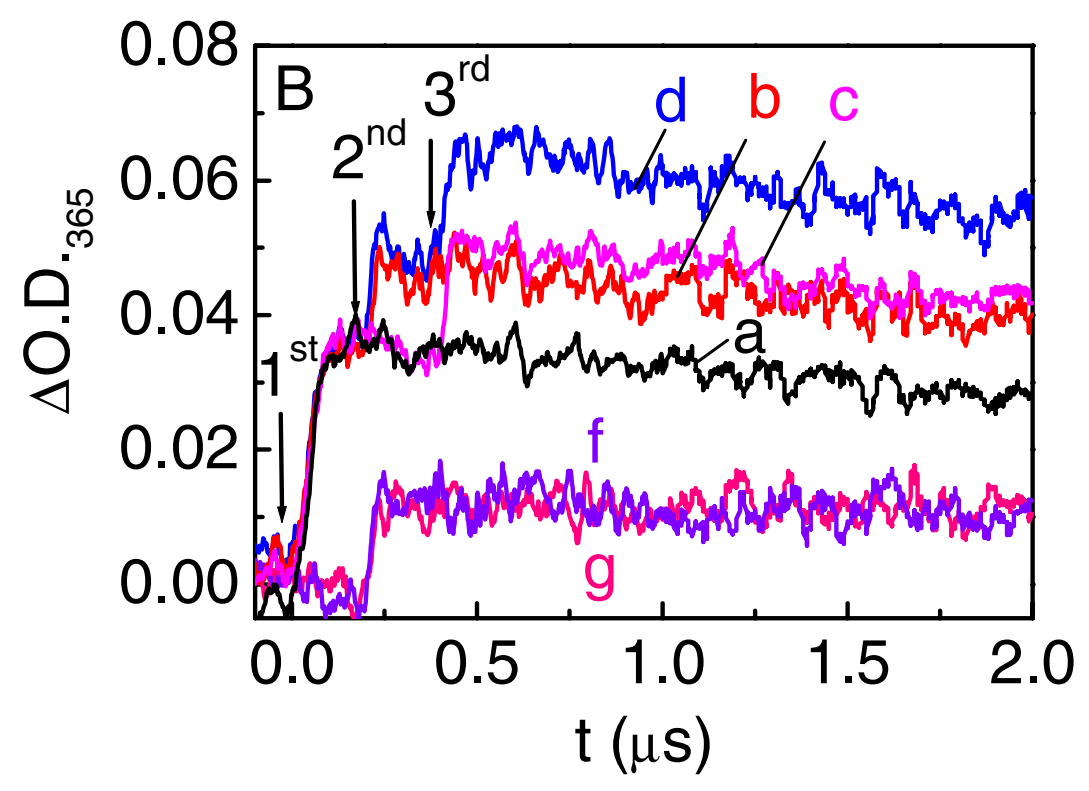

Figure S1 Three-color three-laser photolysis of 1-(BPO-CH $365 \mathrm{~nm}$ ) in Ar-saturated ACN. The transient spectra observed during the irradiation of 308-nm laser (at $500 \mathrm{~ns}$ after the laser pulse) (a), successive irradiation with 308- and 430-nm lasers (at $300 \mathrm{~ns}$ after the second laser pulse; delay time between the two lasers: $200 \mathrm{~ns}$ ) (b), successive irradiation of 308- and 355-nm lasers (at $100 \mathrm{~ns}$ after the second laser pulse; delay time between the two lasers: $400 \mathrm{~ns}$ ) (c), successive irradiation of 308-, 430-, and 355-nm lasers (at $100 \mathrm{~ns}$ after the third laser pulse; delay time between the lasers: $200 \mathrm{~ns}$ and $200 \mathrm{~ns}$ ) (d), the irradiation of 355-nm laser (at $100 \mathrm{~ns}$ after the laser pulse) (e). The inset in A shows the spectra b-a (f) and d-c $(\mathrm{g}) .1^{\text {st }}, 2^{\text {nd }}$, and $3^{\text {rd }}$ in B refer to the irradiation time of 308-, 430-, and 355-nm laser, respectively.

The transient absorption spectrum of $1-\mathrm{NpCH}_{2} \bullet$ was obtained by subtraction of the spectrum 
observed after the first 308-nm laser irradiation (a) from that after the first 308 and second 430-nm laser irradiation (b), (b)-(a). The change of the transient absorption of 1- $\mathrm{NpCH}_{2} \bullet$ during the third 355-nm laser irradiation was obtained after subtraction of the spectrum obtained after the first 308- and third 355-nm laser irradiation (c) from that after the first 308-, second 430-, and third 355-nm laser irradiation (d), (d)-(c). No bleaching of the transient absorption at $365 \mathrm{~nm}$ assigned to $1-\mathrm{NpCH}_{2} \bullet$ was observed during the three-color three-laser photolysis of 1-(BPO- $\left.\mathrm{CH}_{2}\right) \mathrm{Np}$. The third 355-nm laser intensity (10 $\mathrm{mJ}$ pulse ${ }^{-1}$ ) is not enough for two-photon ionization of 1- $\mathrm{NpCH}_{2} \bullet$ to give 1- $\mathrm{NpCH}_{2}{ }^{+}$.

It is known that 1- $\mathrm{NpCH}_{2} \bullet$ will be excited to give 1- $\mathrm{NpCH}_{2} \bullet\left(\mathrm{D}_{\mathrm{n}}\right)$ with lifetime of several tens ns under the third 355-nm laser irradiation. ${ }^{1-3}$ No bleaching of the transient absorption at $365 \mathrm{~nm}$ during the three-color three-laser photolysis of 1-(BPO-CH $)$ Np suggested that 1- $\mathrm{NpCH}_{2} \bullet\left(\mathrm{D}_{\mathrm{n}}\right)$ decays to reproduce 1- $-\mathrm{NpCH}_{2} \bullet$. No reaction occurred from 1- $-\mathrm{NpCH}_{2} \bullet\left(\mathrm{D}_{\mathrm{n}}\right)$.

\section{Three-color three-laser flash photolysis of 1,4-(BPO- $\left.\mathrm{CH}_{2}\right)_{2} \mathrm{~Np}$}

Similar experimental results to those of $1,8-\left(\mathrm{BPO}-\mathrm{CH}_{2}\right)_{2} \mathrm{~Np}$ were obtained for $1,4-\left(\mathrm{BPO}-\mathrm{CH}_{2}\right)_{2} \mathrm{~Np}$ during the three-color three-laser photolysis of 1,4-(BPO-CH$)_{2} \mathrm{~Np}$. The results are shown in Figure S2

\section{A, B.}



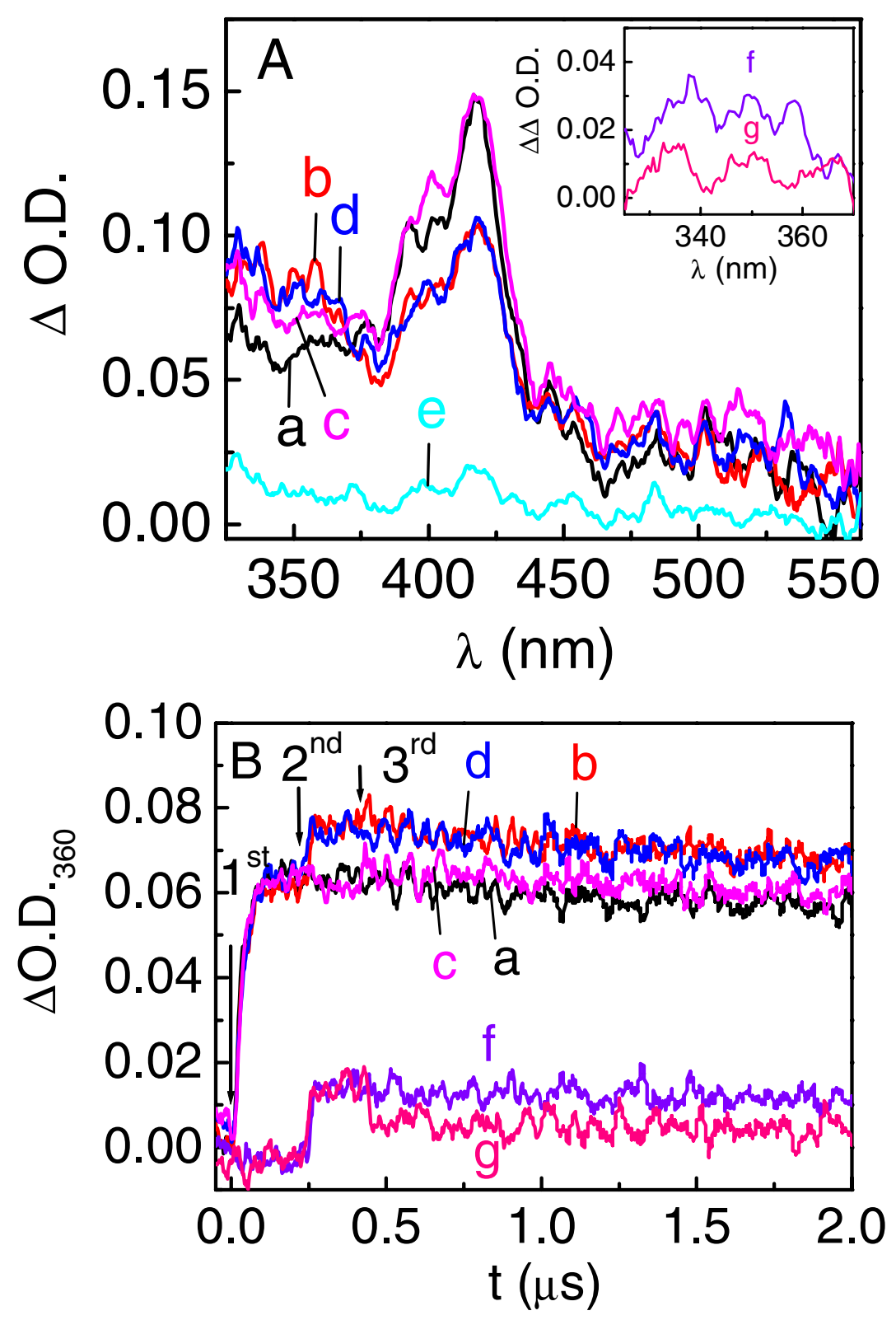

Figure S2 Three-color three-laser photolysis of 1,4-(BPO- $\left.\mathrm{CH}_{2}\right)_{2} \mathrm{~Np}$ (A, spectra; B, time profiles detected at $360 \mathrm{~nm}$ ) in Ar-saturated $\mathrm{ACN}$. The transient spectra observed during the irradiation of 308-nm laser (at $500 \mathrm{~ns}$ after the laser pulse) (a), successive irradiation with 308- and 430-nm lasers (at $300 \mathrm{~ns}$ after the second laser pulse; delay time between the two lasers: $200 \mathrm{~ns}$ ) (b), successive irradiation of 308- and 355-nm lasers (at $100 \mathrm{~ns}$ after the second laser pulse; delay time between the two lasers: $400 \mathrm{~ns}$ ) (c), 
successive irradiation of 308-, 430-, and 355-nm lasers (at $100 \mathrm{~ns}$ after the third laser pulse; delay time between the lasers: $200 \mathrm{~ns}$ and $200 \mathrm{~ns}$ ) (d), the irradiation of 355-nm laser (at $100 \mathrm{~ns}$ after the laser pulse) (e). The inset in A shows the spectra b-a (f) and d-c (g). $1^{\text {st }}, 2^{\text {nd }}$, and $3^{\text {rd }}$ in B refer to the irradiation time of 308-, 430-, and 355-nm laser, respectively.

\section{References}

(1) Johnston, L. J.; Scaiano, J. C. J. Am. Chem. Soc. 1985, 107, 6368.

(2) Johnston, L. J. Chem. Rev. 1993, 93, 251.

(3) Adam, W.; Schneider, K.; Steenken, S. J. Org. Chem. 1997, 62, 3727. 